\section{PSICOLOGIA IBEROAMERICANA}

\section{Psicología lberoamericana}

ISSN: 1405-0943

psicología.iberoamericana@uia.mx

Universidad Iberoamericana, Ciudad de

México

México

Córdova Osnaya, Martha; Rosales Pérez, José Carlos; Reyes Macías, Gisell Stephania Ideación suicida en estudiantes de la Universidad Tecnológica de Costa Grande, Guerrero (México)

Psicología Iberoamericana, vol. 21, núm. 2, julio-diciembre, 2013, pp. 38-47

Universidad Iberoamericana, Ciudad de México

Distrito Federal, México

Disponible en: http://www.redalyc.org/articulo.oa?id=133930525005

Cómo citar el artículo

- Número completo

- Más información del artículo

- Página de la revista en redalyc.org

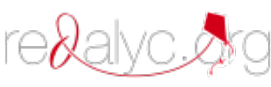

Sistema de Información Científica

Red de Revistas Científicas de América Latina, el Caribe, España y Portugal

Proyecto académico sin fines de lucro, desarrollado bajo la iniciativa de acceso abierto 


\title{
Ideación suicida en estudiantes de la Universidad Tecnológica de Costa Grande, Guerrero (México)
}

\author{
Suicidal ideation in students of the Technological University \\ of Costa Grande Guerrero (Mexico)
}

\author{
Martha Córdova Osnaya*1 \\ José Carlos Rosales Pérez** \\ Gisell Stephania Reyes Macías*** \\ Facultad de Estudios Superiores IZTACALA \\ Universidad Nacional Autónoma de México
}

\section{RESUMEN}

El objetivo fue registrar la presencia de ideación suicida e identificar su relación con variables de identificaciones personales y psicológicas. Participaron 449 estudiantes: 307 hombres (68.4\%) y 142 mujeres (31.6\%) de la Universidad Tecnológica del Estado de Guerrero, elegidos en forma no aleatoria. Se aplicó la Escala de Detección de Ideación Suicida en jóvenes, integrada por un cuestionario de datos personales, cinco escalas de constructos psicológicos y la Escala de Ideación Suicida de Roberts-Ces-D. Se obtuvo el modelo de explicación de ideación suicida por sexo y por conjunto de variables, que señala, en ambos sexos, al antecedente de intento suicida y la emoción negativa como las principales variables explicativas. Se observó un mayor número de variables en el modelo de mujeres que en el de hombres. Finalmente, se discute la relevancia de lo encontrado y su relación con el comportamiento suicida.

Palabras clave: ideación suicida, jóvenes, escalas.

\section{ABSTRACT}

This article seeks to document the presence of suicidal ideation and to identify its relation with personal identification and psychological variables. A non-randomized process was used select the 449 student participants: 307 men (68.4\%) and 142 women (31.6\%) from the Technological University of the state of Guerrero. The Scale for Suicide Ideation for young people was applied, involving a personal information questionnaire, five scales of psychological constructs and the Roberts-CES-D suicide ideation scale. The suicide ideation model was obtained according to sex and to a series of variables. We obtained a model that indicates, in both sexes, the background of attempted suicide and negative emotions as the main explanatory variables. We observed a greater number of variables in the women's model than the men's model. Finally, we examined the relevance of the findings and their relation to suicidal behavior.

Keywords: suicidal ideation, young people, scales.

\footnotetext{
${ }^{1}$ Investigación realizada gracias al Programa de Apoyo a Proyectos de Investigación e Innovación Tecnológica (PAPIIT) de la UNAM núm. IN301410, cuyo nombre es "Estimación de la incidencia de la ideación suicida y su asociación jerárquica con variables psicológicas y sociodemográficas en jóvenes universitarios de la Región Centro y Centro Sur de la República Mexicana".

Los autores agradecen a las autoridades de la Universidad Tecnológica de Costa Grande, Guerrero, y a Diana Noemí Rosales Lobato por el apoyo recibido para la aplicación del instrumento.

Recibido: 19 de abril, 2013 - Aceptado: 24 de agosto, 2013.

${ }^{*}$ Profesora titular de la Licenciatura en Psicología de la Facultad de Estudios Superiores Iztacala. Tel. 587147 71. mcordova@campus.iztacala.unam.mx **Profesor titular de la Carrera de Psicología de la Facultad de Estudios Superiores Iztacala. Tel. móvil 55852715 73. jcrosales@campus.iztacala. unam.mx

***Psicóloga. Tel. 530500 70. zthar.0_o@hotmail.com

Correspondencia: Carrera de Psicología. Facultad de Estudios Superiores Iztacala, Av. De los Barrios 1, col. Los Reyes Iztacala, Tlalnepantla, Estado de México, C. P. 54090.
} 


\section{INTRODUCCIÓN}

Según la Organización Mundial de la Salud (oms), el suicidio se ha convertido en un importante problema de salud mundial, especialmente en jóvenes (oms, 2006). A pesar de que México se encuentra entre los países con menor tasa de suicidio (<6.5) (ops, 2005), las estadísticas señalan que en adolescentes y jóvenes de quince a veintinueve años — de 1970 al 2007-éste se ha incrementado en 275\% (Borges, Orozco, Benjet \& Medina-Mora, 2010). Desde 1980, las lesiones intencionales se han situado como la segunda causa de muerte en varones y la tercera en mujeres de quince a veinticuatro años (Conapo, 2009). Además, desde 2001, el suicidio representa $50 \%$ de las muertes violentas en la población de la misma edad (INEGI, 2002; 2004; 2007; 2011). La investigación del suicidio en jóvenes es prioritaria para generar estrategias de prevención y atención de los casos detectados con riesgo.

\section{Investigación del comportamiento suicida}

El comportamiento suicida se identifica con tres componentes que pueden o no ser secuenciales; ideación suicida, intento suicida y suicidio consumado (Pokorny, 1974; Brown, Jeglinc, Henriques \& Beck, 2008). A partir de lo anterior se definen tres poblaciones de estudio. La primera se conforma por los ideadores suicidas; en ésta los sujetos piensan, desean y planean cometer suicidio pero no han efectuado un intento. La segunda corresponde a los intentadores suicidas, quienes llevaron a cabo un acto de autolesión con el propósito de finalizar con su vida pero no lo lograron. A la última población pertenecen los consumadores del suicidio, que se caracterizan por realizar un acto de autolesión con el fin de terminar con su vida y logran su cometido (Beck, Weissman, Lester \& Trexler, 1976). En razón del mayor riesgo implicado, la investigación del suicidio se ha orientado, principalmente, al estudio de los consumadores y de los intentadores. No obstante, el estudio de los ideadores plantea la posibilidad de obtener información sustantiva para la comprensión del comportamiento antes de la ocurrencia de un intento o suicidio consumado, lo que puede ser significativo para su prevención (Pérez \& Moskera, 2006).

\section{Presencia de ideación suicida en universitarios mexicanos}

Para el estudio de la presencia de ideación suicida en estudiantes universitarios mexicanos se ha reportado el empleo de distintos instrumentos y criterios. El instrumento que se ha aplicado con mayor frecuencia es la Escala de Ideación Suicida de Roberts CES-D. Con su aplicación y con criterio de un puntaje $\geq 1 \mathrm{DE}+\bar{X}$, se ha informado que en universitarios del estado de Morelos se identificó presencia de ideación suicida en $8.4 \%$ de los hombres y en $11.3 \%$ de las mujeres (Rosales, Córdova \& Ramos, 2012); asimismo, en alumnos del Estado de México se registró esta presencia en 13.4\% de los hombres y en $13.1 \%$ de las mujeres (Rosales \& Córdova, 2011). También con el uso de la Escala de Ideación Suicida de Roberts CES-D, pero con criterio de respuesta positiva al menos a uno de los reactivos, se informó que en universitarios del Distrito Federal se observó presencia de ideación suicida en 30\% de los hombres y en $27 \%$ de las mujeres (González-Forteza, García, Medina-Mora \& Sánchez, 1998). Igualmente, con el empleo de la Escala de Ideación Suicida de Roberts CES-D pero con criterio de reporte por promedio del puntaje en la escala, en estudiantes del Estado de México se obtuvo un promedio de 1.47\% en hombres y de $1.78 \%$ en mujeres (Rosales, Córdova \& Villafaña, 2011). El siguiente instrumento que se ha reportado para el registro de la presencia de ideación suicida en jóvenes universitarios corresponde al uso de preguntas específicas. Así, se informó que en alumnos de Saltillo, Coahuila, 22\% de la muestra total respondió afirmativamente a la pregunta “ ¿Ha pensado seriamente en cometer suicidio?" (Carrillo, Valdez, Vázquez, Franco \& De la Peña, 2010). También, en universitarios del estado de Tlaxcala, 5.2\% de los hombres y $7.8 \%$ de las mujeres respondieron "casi siempre" y "todo el tiempo" al reactivo "He pensado en suicidarme" (Córdova, Eguiluz \& Rosales, 2011). Asimismo, en estudiantes del Distrito Federal, 3.5\% de la muestra total contestó positivamente a los reactivos "Sabe si alguien de su familia ha intentado suicidarse alguna vez?” y ¿Ha pensado alguna vez en suicidarse?” (Lazarevich, Delgadillo, Rodríguez \& Mora, 2009). Otro instrumento que se ha aplicado para la detección de ideación suicida en jóvenes universitarios corresponde a la Escala de Ideación Suicida de Beck, Kovacs 
y Weissman (1979). Con este instrumento aplicado a estudiantes del Distrito Federal se informó que 59.9\% de los jóvenes respondieron positivamente al menos a un reactivo de la escala (Córdova, Rosales, Caballero \& Rosales, 2007). También con esta escala, aplicada a universitarios del Distrito Federal, se notificó que 16\% respondió positivamente al menos a tres reactivos de la escala (González, Díaz, Ortiz, González-Forteza \& González, 2000). Finalmente, los instrumentos que se identifican con un solo reporte de investigación son los siguientes. Con aplicación del Symptom Check List (sCL-90) a universitarios del Distrito Federal y con criterio del promedio en el puntaje de la escala, se informó de un promedio de $0.2 \%$ (Heinze, Vargas \& Cortés, 2008). Con el Cuestionario General de Salud (GHQ) aplicado a universitarios del Distrito Federal y con criterio del promedio del puntaje en la escala, se notificó un promedio de 1.4\% (Romero \& Medina-Mora, 1987). Con aplicación del Inventario de Orientaciones Suicidas de Casullo a estudiantes de la zona metropolitana del Distrito Federal y con criterio de un puntaje correspondiente a los niveles de ideación suicida conforme a la escala, se informó que 32.2\% de los hombres y $42.6 \%$ de la mujeres presentaron ideación suicida grave (Coffin, Álvarez \& Marín, 2011).

Estos datos advierten la presencia de ideación suicida en jóvenes universitarios, con una mínima tendencia de que ésta sea mayor en mujeres que en hombres. La variabilidad entre los reportes fue alta: desde $59.9 \%$ hasta $3.5 \%$ en la muestra total; desde $5.2 \%$ hasta $13.4 \%$ en hombres, y desde $7.8 \%$ hasta $13.1 \%$ en mujeres, además de gran diversidad de instrumentos y procedimientos empleados para su registro.

\section{VARIABLES CORRELACIONADAS CON LA PRESENCIA DE IDEACIÓN SUICIDA EN JÓVENES UNIVERSITARIOS}

Un aspecto central de la identificación de la presencia de ideación suicida corresponde al reconocimiento de las variables relacionadas con ella. En estudiantes universitarios mexicanos, las variables asociadas a la presencia de ideación suicida se agrupan en seis categorías. La primera corresponde a problemas familiares: mala relación con el padre (González-Forteza et al., 1998), respuestas de afrontamiento agresivas con la familia (González-Forteza et al., 1998), problemas con la familia (Lazarevich et al., 2009), condición económica insuficiente en la familia (Rosales \& Córdova, 2011) y disciplina en la infancia (Rosales \& Córdova, 2011). La segunda se refiere a antecedentes de experiencias personales negativas: abuso sexual (Rosales et al., 2011); situaciones humillantes (Rosales et al., 2011) y experiencias lamentables (Rosales \& Córdova, 2011). La tercera concierne a problemas escolares: académicos (Lazarevich et al., 2009) y de calificación baja (Rosales et al., 2011). La cuarta corresponde a condiciones personales negativas: baja autoestima, pensamientos negativos o ambivalentes sobre sí mismo (Córdova et al., 2007), ansiedad, impulsividad y rasgos depresivos (Lazarevich et al., 2009). La quinta hace referencia al consumo de sustancias: de drogas (Lazarevich et al., 2009) y de alguna otra para sentirse bien (Rosales \& Córdova, 2011). La sexta se enfoca en el comportamiento suicida: intento de suicidio de alguna persona cercana (Rosales \& Córdova, 2011) y antecedente de intento de suicidio (Rosales et al., 2011).

Dicha información sitúa a los problemas familiares, los antecedentes de experiencias personales negativas y los problemas escolares como las condiciones relacionadas, principalmente, con la presencia de ideación suicida en jóvenes universitarios.

El objetivo de esta investigación fue registrar esta presencia e identificar las variables correlacionadas con este pensamiento en una muestra de jóvenes universitarios de la Universidad Tecnológica de Costa Grande, Guerrero, México. El empleo de la Escala de Ideación Suicida de Roberts-CES-D se debió a que permite registrar su presencia por medio de síntomas (síntoma igual a reactivo) y, por lo tanto, de la puntuación total de la escala. Además, es la escala que más se utiliza para este propósito en jóvenes universitarios mexicanos, lo que posibilitaría contrastar los resultados encontrados en otras investigaciones. Finalmente, el estudio se llevó a cabo con estudiantes del estado de Guerrero porque es una región de la cual no se tiene información acerca de la presencia de ideación suicida en jóvenes universitarios. 


\section{MÉTODO}

\section{Participantes}

La muestra fue no aleatoria e intencional, de la Universidad Tecnológica de Costa Grande. Estuvo constituida, inicialmente, por 464 estudiantes, que correspondió a 36\% de la población estudiantil. Se aplicaron tres elementos de exclusión: no cumplir el criterio de tener al menos $80 \%$ de respuestas en el instrumento, mencionar que se estaba bajo tratamiento psicológico o psiquiátrico, y registrar una edad mayor a veintisiete años. Aplicados los criterios de exclusión, la muestra de estudio quedó integrada por 449 estudiantes: 307 hombres $(68.4 \%)$ y 142 mujeres $(31.6 \%)$ con un promedio de edad de 19.36 años y $\mathrm{DE}=1.41$.

\section{Instrumentos}

Se utilizó el instrumento "Detección de ideación suicida en jóvenes", estructurado por el grupo de investigación perteneciente al proyecto "Evaluación de la ideación suicida en jóvenes", del programa de investigación de la Facultad de Estudios Superiores Iztacala, de la Universidad Nacional Autónoma de México (UNAM). Está integrado por tres secciones.

La sección I es un cuestionario de datos personales. Está conformado por 26 preguntas (23 cerradas, tres abiertas) generales y específicas. Las generales corresponden a edad, sexo y estado civil. Las específicas hacen referencia a información de asuntos escolares (promedio de calificación y percepción de desempeño académico), experiencias negativas (abuso sexual, víctima de situaciones humillantes, reporte de experiencias lamentables, muerte de alguna persona cercana importante, si alguna persona cercana se ha suicidado o intentado suicidarse, intento de suicidio y número de intentos), familiares (condición económica de la familia y disciplina ejercida durante la infancia) o condiciones personales (religión, si el dinero con el que cuenta es suficiente, costumbre de consumir algo para sentirse bien, si se percibe agresivo, si tiene ideas persistentes que no puede evitar, si ha recibido atención psicológica o psiquiátrica, y si actualmente se encuentra en tratamiento psicológico o psiquiátrico).

La sección II se compone de cinco escalas, cuyo número de reactivos responde al análisis de confiabilidad y validez factorial en la integración del instru- mento para la detección de ideación suicida en jóvenes (Rosales, 2012). En orden, las escalas son:

- Escala de Actitud Disfuncional: cuenta con 21 reactivos de los cuarenta de la Escala de Actitudes Disfuncionales (DAS) de Weissman y Beck (1978) (Sanz \& Vázquez, 1993). El alfa de Cronbach fue de 0.80 , agrupación de reactivos en tres factores y varianza explicada total de $45 \%$.

- Escala de Locus de Control: son siete reactivos de los ocho de la Escala de Locus de Control de González-Forteza (1992), con alfa de Cronbach de 0.70 , agrupación de reactivos en dos factores y varianza explicada total de $55 \%$.

- Escala de Apoyo Social Percibido: se conforma por seis reactivos de los 12 de la Escala de Apoyo Social Percibido de Zimet, Dahlem y Farley (1988), con alfa de Cronbach de 0.80, agrupación de reactivos en dos factores y varianza explicada total de $75 \%$.

- Escala de Desesperanza: ocho reactivos de los veinte de la Escala de Desesperanza de Beck (Beck et al., 1974), con alfa de Cronbach de 0.80, agrupación de reactivos en dos factores y varianza explicada total de $46 \%$.

- Escala de Trastornos Emocionales: es el total de reactivos de la Escala de Trastornos Emocionales de Berwick, Murphy, Goldamn, Ware, Barsky \& Weinstein (1991) (cinco reactivos), con alfa de Cronbach de 0.79, agrupación de reactivos en un solo factor y varianza explicada total de $54 \%$.

La sección III incorpora la Escala de Ideación Suicida de Roberts-CES-D, conformada por cuatro reactivos. El primero, "No podía seguir adelante", pertenece a la Escala para la Depresión del Centro de Estudios Epidemiológicos (CES-D) adaptada por Roberts para adolescentes (Radloff, 1977). Los otros tres reactivos son de la Escala de Ideación Suicida de Roberts y Chen (1995): “Tenía pensamientos sobre la muerte, sentía que mi familia estaría mejor si yo estuviera muerto(a) y pensé en matarme". Cuenta con registro de alfa de Cronbach de 0.65 a 0.81 y agrupación de reactivos en un solo factor, con varianza explicada total de $52.25 \%$ a 68.6\% (González-Forteza et al., 1998; Rosales \& Córdova, 2011; Rosales et al., 2011; Rosales et al., 2012; 
Unikel \& Gómez-Peresmitré, 2004). Dos preguntas se refieren a algún antecedente de intento suicida, una a su ocurrencia y otra a su frecuencia.

La opción de respuesta para los reactivos de las escalas de la sección II, con excepción de la Escala de Trastorno Emocional, correspondió a un formato tipo Likert con cuatro opciones: "Totalmente de acuerdo", "De acuerdo", "En desacuerdo" o "Totalmente en desacuerdo". El puntaje de respuesta fue de uno a cuatro, donde el valor más alto se asignó a la opción que definía una respuesta que afirmara la presencia de algún síntoma que pudiera corresponder a ideación suicida. Por ejemplo, en el reactivo uno de la Escala de Actitud Disfuncional "Si desagrado a los demás no puedo ser feliz", el valor cuatro corresponde a la opción "Totalmente de acuerdo". En la Escala de Trastorno Emocional, hubo cinco opciones de respuesta; 0\%, $20 \%, 40 \%, 60 \%, 80 \%$ y $100 \%$. Éstas eran relativas al porcentaje del tiempo. En ellas se señalaba cómo se sintió el estudiante durante el mes anterior a la aplicación, de acuerdo con lo que se especificaba en cada reactivo. En la Escala de Ideación Suicida había cuatro opciones de respuesta; 0 días, 1-2 días, 3-4 días o 5-7 días, correspondientes a la duración del pensamiento la semana anterior al registro.

\section{Variables}

La variable criterio se identifica con ideación suicida, y refiere a los pensamientos que tuvieron los sujetos la semana anterior a la aplicación acerca del deseo de morir, de quitarse la vida y de la forma de llevar a cabo el acto suicida, con criterio de un puntaje $\geq \mathrm{a} 1 \mathrm{DE}+\bar{X}$ en la Escala de Ideación Suicida de Roberts-CES-D para referir la presencia de la ideación suicida.

Las variables predictoras son: a) datos de identificación personal: características personales que se han detectado asociadas a la presencia de ideación suicida en jóvenes, con cambio de las respuestas en variables dummy. El valor uno se asignó a la categoría que regularmente se asocia a la presencia de ideación suicida y el valor cero a las categorías restantes de cada variable; b) constructos psicológicos: se registró el valor de la puntuación total que refería el grado de identificación con el conjunto de afirmaciones de cada escala; c) actitud disfuncional: son declaraciones funcionales y disfuncionales referentes a la aceptación personal, el éxito y sentirse cómodo(a); d) locus de control: aseveraciones acerca de si el control de los éxitos o fracasos personales es externo o interno; e) apoyo social: son enunciados referentes a si se recibe apoyo de la familia y los amigos; f) desesperanza: se refiere a proposiciones sobre la percepción positiva o negativa del futuro; g) estado emocional: afirmaciones acerca de sentir nerviosismo, tristeza, abatimiento, felicidad o calma, el mes anterior a la aplicación.

\section{Procedimiento}

- Aplicación en los salones de clase de la Universidad Tecnológica de Costa Grande, donde el encuestador fue presentado por un representante de la institución. A continuación éste recordaba el propósito del estudio, su derecho a no participar y que incluso si habían aceptado podían declinar en cualquier momento.

- Realizadas las aplicaciones, se efectuó el vaciado de la información en una base de datos del Programa SPSS versión 18, para proceder a la verificación, la corrección y la selección de datos conforme a los criterios de exclusión.

- Se obtuvo la fiabilidad y validez factorial del instrumento, con base en el alfa de Cronbach y del análisis factorial por componentes principales, con rotación varimax.

- Se registró la presencia de ideación suicida en hombres y mujeres, con base en el criterio de un puntaje en la escala de ideación suicida $\geq \mathrm{a} 1 \mathrm{DE}+\overline{\mathrm{X}}$

- Se corrió un modelo de regresión logística binaria por el método introducir, para identificar la presencia de ideación suicida, a partir del conjunto de variables psicológicas y de identificación personal.

\section{RESULTADOS}

\section{Confiabilidad y validez factorial}

La confiabilidad de las escalas de constructos psicológicos en hombres fue igual a 0.77 y 0.70 en mujeres, con varianza explicada total de $62.89 \%$ y $68.24 \%$. Con respecto a la Escala de Ideación Suicida de RobertsCES-D, la fiabilidad registrada en hombres fue igual a 0.77 y 0.75 en mujeres. El registro de validez factorial de los constructos psicológicos, con base en el análisis 
factorial con rotación varimax y criterio de carga factorial $\geq$ a 0.40 para la permanencia del reactivo, señaló una organización de reactivos en factores correspondientes a cada escala. En la tabla 1 se indica el número de reactivos que permanecieron y la varianza explicada por factor, donde se observa que el factor con mayor varianza explicada para los hombres fue locus de control interno, con $8.72 \%$, en tanto que para las mujeres fue exigencia de aprobación, con $11.86 \%$.

Con respecto a la Escala de Ideación Suicida, se encontró que los reactivos se agruparon en un solo factor tanto en hombres como en mujeres, con varianza explicada total de $59.83 \%$ para ambos géneros.

\section{Promedio y punto de corte para escala de ideación suicida}

En hombres se registró $\bar{X}=1.53$ con $\mathrm{DE}=3.11$ y en mujeres $\bar{X}=2.06$ con $\mathrm{DE}=3.32$. Esto definió, conforme al criterio de una puntuación $\geq$ a $1 \mathrm{DE}+\bar{X}$ para el establecimiento del punto de corte, un puntaje de cinco para la presencia de ideación suicida.

En hombres, el porcentaje de la presencia de ideación suicida fue de $11.7 \%$ (36), en tanto que en mujeres fue de $18.3 \%$ (26).

\section{Modelo de explicación de ideación suicida con variables de identificación personal, por sexo}

En hombres se reconocieron tres variables que aumentan la probabilidad de la presencia de ideación suicida: antecedente de intento suicida, vivencia de situaciones humillantes y considerarse agresivo; $x_{(3)}^{2}=45.05$, $\mathrm{p}<0.001$, varianza explicada del $26.5 \%$ y porcentaje de pronóstico de ideación suicida de $27.8 \%$, donde la variable con mayor peso fue antecedente de intento suicida (véase tabla 2).

Tabla 1. Número de reactivos y valores por sexo, resultantes del análisis psicométrico en las escalas psicológicas del instrumento para detección de la ideación suicida

\begin{tabular}{|c|c|c|c|c|c|c|}
\hline \multirow[b]{2}{*}{$\begin{array}{l}\text { Escalas de Constructos } \\
\text { Psicológicos }\end{array}$} & \multicolumn{3}{|c|}{ Hombres } & \multicolumn{3}{|c|}{ Mujeres } \\
\hline & Nombre del factor & N. R. & $\begin{array}{c}\text { Varianza } \\
\text { explicada } \\
(\%)\end{array}$ & Nombre del factor & N. R. & $\begin{array}{c}\text { Varianza } \\
\text { explicada } \\
(\%)\end{array}$ \\
\hline \multirow{2}{*}{$\begin{array}{l}\text { Escala de Actitud } \\
\text { Disfuncional }\end{array}$} & Exigencia de comodidad & 5 & 7.75 & & & \\
\hline & Exigencia de aprobación & 4 & 7.75 & $\begin{array}{l}\text { Exigencia de } \\
\text { aprobación }\end{array}$ & 5 & 11.86 \\
\hline $\begin{array}{l}\text { Escala de Locus de } \\
\text { Control }\end{array}$ & Interno & 3 & 8.72 & Interno & 3 & 10.58 \\
\hline \multirow{2}{*}{$\begin{array}{l}\text { Escala de Apoyo Social } \\
\text { Percibido }\end{array}$} & Apoyo familia & 3 & 7.56 & Apoyo familia & 3 & 10.90 \\
\hline & Apoyo amigos & 3 & 8.51 & Apoyo amigos & 3 & 11.64 \\
\hline Escala de Desesperanza & Futuro negativo & 4 & 8.42 & Futuro negativo & 3 & 11.80 \\
\hline $\begin{array}{l}\text { Escala de Trastornos } \\
\text { Emocionales }\end{array}$ & Emoción negativa & 3 & 8.07 & Emoción negativa & 3 & 11.43 \\
\hline
\end{tabular}

Nota: N. R. = Número de reactivos en el factor. 
En mujeres se ubicaron cinco variables que explican la presencia de ideación suicida y cuatro que incrementan su probabilidad: antecedente de intento de suicidio, desempeño académico, abuso sexual e ideas que no puede evitar. Se halló una variable que disminuye la probabilidad de presencia de ideación suicida: promedio de calificación (véase tabla 3).

Modelo de explicación estadística de ideación suicida con variables correspondientes a los constructos psicológicos, por sexo

En hombres se identificó un modelo integrado por una variable, emoción negativa, que incrementa la proba- bilidad de presencia de ideación suicida; $x_{(1)}^{2}=33.72$ $\mathrm{p}<0.001$, varianza explicada de $20.2 \%$ y pronóstico de $8.3 \%$. En mujeres, se halló un modelo compuesto por dos variables: emoción negativa, que incrementa la probabilidad de presencia de ideación suicida, y apoyo familiar, que disminuye la probabilidad de presencia de ideación suicida; $x^{2}{ }_{(2)}=36.44 \mathrm{p}<0.001$. La varianza explicada fue de $36.9 \%$ y el pronóstico de $38.5 \%$. Estos datos señalan que la emoción negativa es la variable psicológica que explica la ideación suicida en ambos sexos, y que en mujeres es significativa la percepción de apoyo familiar, para disminuir la probabilidad de presencia de ideación suicida.

Tabla 2. Modelo de regresión logística binaria; variables de identificación personal en hombres.

\begin{tabular}{|c|c|c|c|c|c|c|c|}
\hline \multirow[b]{2}{*}{ Variables } & \multirow[b]{2}{*}{ Parámetros } & \multirow[b]{2}{*}{ Error } & \multirow[b]{2}{*}{ Wald } & \multirow[b]{2}{*}{ gl } & \multirow[b]{2}{*}{ Exp. (B) } & \multicolumn{2}{|c|}{ Intervalos al $95 \%$} \\
\hline & & & & & & Inferior & Superior \\
\hline Intentos de suicidio & $2.06^{*}$ & 0.57 & 12.91 & 1 & 10.68 & 2.55 & 24.33 \\
\hline V. Situaciones humillantes & $1.16 * *$ & 0.40 & 8.18 & 1 & 3.19 & 1.44 & 7.08 \\
\hline Ser agresivo & $0.72 * *$ & 0.22 & 10.06 & & 2.06 & 1.31 & 3.21 \\
\hline
\end{tabular}

Nota: Constante $=-4.45, R^{2}$ de Nagelkerke $=0.265 .{ }^{*} p \leq 0.001 .{ }^{*} p \leq 0.01$.

Tabla 3. Modelo de regresión logística binaria; variables de identificación personal en mujeres.

\begin{tabular}{|c|c|c|c|c|c|c|c|}
\hline \multirow{2}{*}{$\begin{array}{l}\text { Valores de las variables } \\
\text { explicativas }\end{array}$} & \multirow[b]{2}{*}{ Parámetros } & \multirow[b]{2}{*}{ Error } & \multirow[b]{2}{*}{ Wald } & \multirow[b]{2}{*}{ gl } & \multirow[b]{2}{*}{ Exp.(B) } & \multicolumn{2}{|c|}{ Intervalos al $95 \%$} \\
\hline & & & & & & Inferior & Superior \\
\hline Intentos de suicidio & $2.43^{*}$ & 0.67 & 12.92 & 1 & 11.46 & 3.03 & 43.36 \\
\hline Desempeño académico & $2.16^{*}$ & 1.03 & 4.33 & 1 & 8.67 & 1.13 & 66.27 \\
\hline Abuso sexual & $1.55^{*}$ & 0.65 & 5.71 & 1 & 4.75 & 1.32 & 17.08 \\
\hline Ideas que no puede evitar & $0.89 *$ & 0.34 & 6.60 & 1 & 2.43 & 1.23 & 4.18 \\
\hline Promedio calificaciones & $-1.54^{*}$ & 0.52 & 8.73 & 1 & 0.21 & 0.07 & 0.59 \\
\hline
\end{tabular}

Nota: Constante $=7.32, \mathrm{R} 2$ de Nagelkerke $=0.559 .{ }^{*} \mathrm{p} \leq 0.05$. 


\section{DISCUSIÓN}

La presencia de ideación suicida identificada en esta investigación $-11.7 \%$ en hombres y $18.3 \%$ en mujeres - fue similar a lo reportado en estudiantes universitarios del Estado de México (13\%) (Rosales \& Córdova, 2011), y en estudiantes universitarias del Distrito Federal (16\%) (González et al., 2000), pero mayor a lo reportado en población abierta de dieciocho a veintinueve años (8.74\%) (Borges et al., 2010). Es necesario considerar que sólo en la investigación de Rosales y Córdova (2011) y en el presente estudio se reporta la misma escala, con el mismo punto de corte, ya que en el trabajo de Borges et al. (2010) se emplean tres preguntas para registrar la presencia de ideación suicida en los últimos doce meses; “¿Ha experimentado la sensación de que no vale la pena vivir?”, “ ¿Ha vivido situaciones ante las que ha deseado dejar de existir?”, “¿Ha pensado que vale más morir que vivir?”, mientras que en el estudio de González et al. (2000) se informa del uso de la Escala de Beck, sin indicación del punto de corte, ni de la temporalidad de la respuesta. Aun con estas diferencias, la información publicada acerca de la presencia de ideación suicida en jóvenes universitarios plantea que esta condición se presenta en un porcentaje que oscila entre $11 \%$ y $18 \%$, con mayor tendencia en mujeres que en hombres.

Con respecto a la explicación estadística de la presencia de ideación suicida, se distingue lo siguiente. Primero, hay mayor número de variables asociadas a la presencia de ideación suicida en mujeres que en hombres, dato que no se ha reportado en estudios previos, y precisa una condición asociada al sexo, que sería relevante confirmar en próximos estudios. Segundo, se confirma lo reportado por González-Forteza et al. (1998) acerca de la importancia del apoyo familiar en mujeres - condición de protección- y de la agresión en hombres - condición de riesgo-. Tercero, sólo en las mujeres de la muestra es significativa la percepción de la condición académica para la manifestación de la ideación suicida; desempeño académico -condición de riesgo- y promedio -condición de protección-. Cuarto, en ambos sexos el antecedente de intento suicida y el estado emocional negativo son factores de riesgo para la presencia de ideación suicida. Esta información confirma lo ya reportado acerca de la repercusión del antecedente de intento suicida (Unikel, Gómez-Peresmitré \& González-Forteza, 2006; Rosales \& Córdova, 2011; Rosales et al., 2011) y del estado emocional negativo (Coffin et al., 2011), en su manifestación en jóvenes universitarios, que consigna la importancia del registro de la ideación suicida, conjuntamente con el antecedente de comportamiento suicida y el estado emocional negativo, para su detección en jóvenes universitarios. Además, es consistente con la aproximación cognitivo-conductual del estudio del comportamiento suicida, respecto al señalamiento de que el antecedente de intento suicida y el estado emocional negativo son indicadores de mayor riesgo de comportamiento suicida (Rudd, 2000).

Por otra parte, es importante denotar la limitación de no contar con una muestra aleatoria de estudiantes en el presente estudio. Aun con ello, fue posible aportar información relevante respecto a la presencia de ideación suicida y de las condiciones asociadas, en universitarios del estado de Guerrero, con lo que se reconocieron semejanzas, así como diferencias, respecto a lo reportado en universitarios mexicanos con ideación suicida. En suma, esto delinea la posibilidad de distintos porcentajes de presencia de ideación suicida, así como diferentes explicaciones estadísticas en las diferentes poblaciones de universitarios mexicanos.

Esta investigación muestra que la identificación de presencia de ideación suicida y de las variables asociadas puede ser una estrategia viable para el reconocimiento de los jóvenes que podrían estar en riesgo de desarrollar el comportamiento suicida. Además, esto puede incidir en el desarrollo de estrategias de atención psicológica, orientadas tanto a la reducción del riesgo de un intento o suicidio consumado, como en el mejoramiento de la calidad de vida de los jóvenes universitarios mexicanos. • 


\section{REFERENCIAS}

Beck, A. T., Kovacs, M. \& Weissman, M. (1979). Assessment of suicidal intent. The scale for suicide ideation. Journal of Consulting and Clinical Psycho$\log y, 47(2), 343-352$.

Beck, A. T., Weissman, A., Lester, D. \& Trexler, L. (1974). The measurement of pessimism: the hopelessness scale. Journal of Consulting and Clinical Psychology, 42(6), 861-865.

Beck, A. T., Weissman, A., Lester, D. \& Trexler, L. (1976). The classification of suicidal behaviors: two dimensions of suicidal intent. Archives of General Psychiatry, 33, 835-837.

Berwick, D. M., Murphy, J. M., Goldamn, P. A., Ware, J. E., Barsky, J. \& Weinstein, M. C. (1991). Performance of a five-item mental health screening test. Medical Care, 29(2), 169-176.

Borges, G., Nock, M., Medina-Mora, M. E., Benjet, C., Lara, C., Chiu, W. T. \& Kessler, R. (2007). The epidemiology of suicide-related outcomes in Mexico. Suicide \& Life-threatening Behavior, 37(6), 627-640.

Borges, G., Orozco, R., Benjet, C. \& Medina-Mora, M. E. (2010). Suicidio y conductas suicidas en México: retrospectiva y situación actual. Salud Pública de México, 52(4), 292-304.

Bravo, M. C. \& Vaquero J. E. (2012). Descubriendolos tipos de estudio y diseños de investigación en psicología. En Chávez, M. \& Tron, R. (Coords.), Análisis descriptivo en Psicología (pp. 1-17). México: Universidad Nacional Autónoma de México-Facultad de Estudios Superiores Iztacala.

Brown, G. K., Jeglinc, E., Henriques, G. \& Beck, A. T. (2008). Terapia cognitiva, cognición y comportamiento suicida. En Ellis, T. E. (Dir.), Cognición y suicidio: teoría, investigación y terapia (pp. 51-72). México: Manual Moderno.

Carrillo, J., Valdez, L., Vázquez, H., Franco, J. \& De la Peña, A. (2010). Depresión, ideación suicida e insomnio en universitarios de Saltillo, problemas relevantes de salud pública. Revista Mexicana de Neurociencia, 11(1), 30-32.

Coffin, N., Álvarez, M. \& Marín, A. (2011). Depresión e ideación suicida en estudiantes de la FESI: un estudio piloto. Revista Electrónica de Psicología Iztacala, 14(1), 341-354.
Consejo Nacional de la Población (Conapo) (2009). La situación demográfica de México 2008. Recuperado de www.conapo.gob.mx/publicaciones/sdm/sdm2008/ 01.pdf

Córdova, M., Eguiluz, L. \& Rosales, J. C. (2011). Pensamientos suicidas en estudiantes universitarios del Estado de Tlaxcala (México). Enseñanza e Investigación en Psicología, 16(1), 155-164.

Córdova, M., Rosales, M. P., Caballero, R. \& Rosales, J. C. (2007). Ideación suicida en jóvenes universitarios: su asociación con diversos aspectos psicosociodemográficos. Psicología Iberoamericana, 15(2), 17-21.

Cortada, N. \& Carro, J. (1975). Estadística aplicada. Buenos Aires: Universitaria de Buenos Aires.

González, M. S., Díaz, M. A., Ortiz, L. S., GonzálezForteza, C. \& González, N. J. (2000). Características psicométricas de la Escala de Ideación Suicida de Beck (ISB) en estudiantes universitarios de la Ciudad de México. Salud Mental, 23(2), 21-30.

González-Forteza, C. (1992). Estresores psicosociales y respuestas de enfrentamiento en los adolescentes: impacto sobre el estado emocional. (Tesis de maestría). Universidad Nacional Autónoma de México, México. González-Forteza, C., García, G., Medina-Mora, M. E. \& Sánchez, M. A. (1998). Indicadores psicosociales predictores de ideación suicida en dos generaciones de estudiantes universitarios. Salud Mental, 21(3), 1-9.

Heinze, G., Vargas, E. \& Cortés, F. (2008). Síntomas psiquiátricos y rasgos de personalidad en dos grupos opuestos de la Facultad de Medicina de la UnAM. Salud Mental, 31(5), 343-350.

Instituto Nacional de Estadística, Geografía e Informática (INEGI) (2002). Estadística de intentos de suicidio $y$ suicidios. Cuaderno Núm. 8. México: INEGI.

Instituto Nacional de Estadística, Geografía e Informática (INEGI) (2004). Estadística de intentos de suicidio y suicidios. Cuaderno Núm. 10. México: INEGI.

Instituto Nacional de Estadística, Geografía e Informática (INEGI) (2007). Estadísticas de intentos de suicidio y suicidios de los Estados Unidos Mexicanos 2005. Recuperado de http://www.inegi.org.mx/prod_serv/ contenidos/espanol/bvinegi/productos/continuas/ sociales/suicidio/2005bis/suicidios_05.pdf

Instituto Nacional de Estadística y Geografía e Informática (INEGI) (2011). Estadística de suicidios de los Estados Unidos Mexicanos 2009. Recuperado de 
http://www3.inegi.org.mx/sistemas/biblioteca/detalle.aspx? $c=11059 \& u p c=702825002056 \& s=e s t \& \operatorname{tg}=1$ $04 \& \mathrm{f}=2 \& \mathrm{pf}=\mathrm{EncH} \& \mathrm{ef}=00 \& \mathrm{cl}=0$

Lazarevich, I., Delgadillo, J., Rodríguez, J. \& Mora, F. (2009). Indicadores psicosociales de riesgo suicida en los estudiantes universitarios. Psiquis, 18(3), 71-79.

Organización Mundial de la Salud (oмs) (2006). Departamento de Salud Mental y Abuso de Sustancias. Trastornos mentales y cerebrales. Prevención del suicidio. Recurso para consejeros. Recuperado de www.who. int/mental_health/media/counsellors_spanish.pdf

Organización Panamericana de la Salud (ops) (2006). Número de muerte por suicidio supera las causadas conjuntamente por guerras, terrorismo y asesinatos. Comunicado de Prensa. Washington D. C. Recuperado de http://www.paho.org/Spanish/DD/ PIN/ps061010.htm

Mendoza-Nuñez, V. (1998). Diseños de Investigación. Tópicos de investigación y posgrado, 4, 219-222.

Pérez, S. \& Moskera, D. (2006). El suicidio, prevención $y$ manejo: memorias de un curso necesario. Madrid: Pléyades.

Pokorny, A. (1974). A scheme for classifying suicidal behaviors. En Beck, A. T. Resnik, H. \& Lettieri, D. (Eds.), The prediction of suicide (pp. 29-44). Chicago: Charles Press Pubs.

Radloff, L. S. (1977). The CES-D scale: a self-report depression scale for research in the general population. Applied Psychology Measurement, 1, 385-401.

Roberts, R. \& Chen, Y. W. (1995). Depressive symptoms and suicidal ideation among mexican-origin and anglo-adolescents. Journal of the American Academy of Child and Adolescent Psychiatry, 34(1), 81-90.

Romero, M. \& Medina-Mora, M. E. (1987). Validez de una versión del Cuestionario General de Salud, para detectar psicopatología en estudiantes universitarios. Salud Mental V, 10(3), 90-97.

Rosales, J. C. (2012). Ideación suicida en jóvenes mexicanos de educación media superior: propuesta de un modelo descriptivo multivariado en hombres y mujeres. (Tesis de doctorado), Universidad Nacional Autónoma de México, México.

Rosales, J. C. \& Córdova, M. (2011). Ideación suicida y su relación con variables de identificación personal en estudiantes universitarios mexicanos. Revista Intercontinental de Psicología y Educación, 13(2), 9-30.

Rosales, J. C., Córdova, M. \& Ramos, R. (2012). Ideación suicida en estudiantes mexicanos: modelo de relación múltiple con variables de identificación personal. Psicología y Salud, 22(1), 63-74.

Rosales, J. C., Córdova, M. \& Villafaña, A. (2011). Presencia de ideación suicida y su asociación con variables de identificación personal en estudiantes mexicanos. Acta Psiquiátrica y Psicológica de América Latina, 57(2), 91-98.

Rudd, M. (2000). The suicidal mode: a cognitive-behavioral model of suicidality. Suicide \& Life-threatening Behavior, 30(1), 18-33.

Sanz, J. \& Vázquez, C. (1993). Adaptación española de la Escala de Actitudes Disfuncionales (DAS) de Weissman y Beck: propiedades clínicas y psicométricas. Análisis y Modificación de Conducta, 19, 707-750.

Unikel, C. \& Gómez-Peresmitré, G. (2004). Validez de constructo de un instrumento para la detección de factores de riesgo en los trastornos de la conducta alimentaria en mujeres mexicanas. Salud Mental, 27(1), 38-49.

Unikel, C., Gómez-Peresmitré, G. \& González-Forteza, C. (2006). Suicidal behaviour, risky eating behaviours and psychosocial correlates in mexican female students. European Eating Disorders Review, 14, 414-421.

Weissman, M. \& Beck, A. T. (1978). Development and validation of dysfunctional Attitude Scale. Sesión de cartel presentada en el encuentro de la Association for Advancement of Behavior Therapy, Chicago.

Zimet, G., Dahlem, S. \& Farley, G. (1988). Multidimensional scale of perceived social support (MSPss). Journal of Personality Assessment, 52, 30-41. 